\title{
HPLC/MS identification of the polyphenols present in an extract of Myrtus communis L. obtained by supercritical fluid extraction
}

\author{
Identificação por HPLC/MS dos polifenóis presentes num extrato de Myrtus communis L. \\ obtido por extração supercrítica
}

\author{
Paula Pereira ${ }^{1,2}$, M. Conceição Oliveira ${ }^{3}$, M. Gabriela Bernardo Gil' ${ }^{2}$, Maria-João Cebola ${ }^{1,4}$ \\ ${ }^{1}$ CBIOS - Universidade Lusófona's Research Centre for Biosciences and Health \\ Technologies, ULHT, Av. Campo Grande, 376, 1749-024 Lisboa, Portugal \\ ${ }^{2}$ CERENA - Centre for Natural Resources and the Environment, Instituto Superior Técnico, Universidade de Lisboa, Av. \\ Rovisco Pais, 1049-001 Lisboa, Portugal \\ ${ }^{3}$ Centro de Química Estrutural, Complexo Interdisciplinar, Instituto Superior Técnico, ULisboa, Av. Rovisco Pais, 1049-001 \\ Lisboa, Portugal \\ ${ }^{4}$ ENIDH - Escola Superior Náutica Infante D. Henrique, Av. Eng. Boneville Franco, 2770-058 Paço de Arcos, Portugal. \\ Email: mjoaocebola@enautica.pt
}

\begin{abstract}
In this work, we studied an extract obtained by supercritical fluid extraction (SFE) using a simpler method of cosolvent (ethanol) addition. Instead of using a liquid pump, which is the most common process, the ethanol was directly introduced in the extraction cell, immediately after loading the cell with the plant sample. it was our intent to investigate if this change would have any effect in the composition of the extract obtained. The experimental conditions used were: temperature $48^{\circ} \mathrm{C}$, pressure $10 \mathrm{MPa}$, supercritical fluid (SCF) flow rate $130.71 \mathrm{dm} 3 \mathrm{~h}-1(0.238$ $\mathrm{kgh}-1)$ and an ethanol volume of $104 \mathrm{~cm} 3$. The composition of the extract obtained was different from previous tests, and the compounds identified by HPLC-MS were quinic acid, quinic acid 3,5-di-O-gallate, quinic acid 3,4,5-galloyl, myricetin-galactoside gallate, quercetin-galactoside gallate, quercetin, and myricetin-galactosiderhamnoside.
\end{abstract}

Keywords: Myrtle, Myrtus communis L., HPLC-MS, supercritical fluid extraction, ethanol, polyphenols.

\section{Resumo}

Neste trabalho, estudámos um extrato obtido por extração de fluido supercrítica (ESC) usando um método mais simples de adição de co-solvente (etanol). Em vez de usar uma bomba de líquidos, que é o processo mais comum, o etanol foi introduzido diretamente na célula de extração, imediatamente após o carregamento da célula com a amostra de planta. Pretendeu-se investigar se esta alteração teria algum efeito na composição do extrato obtido. As condições experimentais utilizadas foram: temperatura $48{ }^{\circ} \mathrm{C}$, pressão $10 \mathrm{MPa}$, caudal de fluido supercrítico (FSC) 130,71 dm3h-1 (0,238 kgh-1) e um volume de etanol de $104 \mathrm{~cm} 3$. Os compostos identificados por HPLCMS neste extrato foram o ácido quínico, o ácido quínico 3,5-di-O-galato, o ácido 3,4,5-galoilquinico, a miricetinagalactósido-galato, a quercetina-galactósido-galato, a miricetina-galactósido e a quercetina-ramnósido).

Palavras-Chave: Murta, Myrtus Communis L., HPLC-MS, extração supercrítica, etanol, polifenóis 


\section{Introduction}

Plants are an immense source of chemical compounds, many of them with a pharmacological interest. Myrtle (Myrtus Communis L.) is an evergreen shrub that grows spontaneously throughout the Mediterranean region and is part of the traditional Portuguese flora. This plant is mentioned as being of interest for areas ranging from medicine to cosmetics in addition to use in the food industry and in the production of liqueurs with digestive properties. Due to the potential economic interest derived from its interesting properties, myrtle has been for some time a research subject for several authors [1-7]. In previously published studies already published in the literature, we have investigated by HPLC-MS the composition of myrtle's leaf extracts obtained through different types of extraction methods, namely, supercritical fluid extraction, conventional extraction and ultrasound extraction. The results obtained have shown variations in the extract's composition in terms of number and type of compounds. Only myricetin-rhamnoside was present in all the extracts obtained, independent of the extraction method [5-6]. In the extracts obtained by conventional extraction, , gallic acid, ellagic acid, myricetin, quercetin and quercetin-rhamnoside have also been identified, while in the supercritical fluid extraction, myricetin-galactoside and quercetinrhamnoside were identified along with the myricetinrhamnoside. As to the extracts obtained by ultrasound, quercetin-galactoside-galatte, myricetin-galactoside, myricetin-galactoside-galatte and myricetin have also been identified[6].

In the present study, an extract, obtained also by supercritical fluid extraction but using a simpler method for the co-solvent addition, was analysed to investigate if this difference would affect the number and type of extracted compounds. Rather than introducing the ethanol in the extraction vessel progressively, over the duration of the extraction using a liquid pump, the total volume of ethanol was added directly into the extraction vessel after the introduction of the sample.

\section{Material and methods}

\section{Plant Material}

Samples of myrtle leaves were collected from the Sintra area (Portugal). After collection, the plant material was identified and deposited in the Herbarium of the Superior Agronomy Institute of the Technical Universi-

\section{Introdução}

As plantas são uma fonte imensurável de compostos químicos, muitos com interesse farmacológico. A murta (Myrtus Communis L.) é um arbusto perene que cresce espontaneamente em toda a região mediterrânica, fazendo parte da flora tradicional portuguesa. Esta planta é referenciada como de interesse em áreas desde a medicina aos cosméticos, tendo também vindo a ser aplicada na indústria alimentar e na produção de licores com propriedades digestivas. Devido ao potencial interesse económico, derivado do seu interessante conjunto de propriedades, a murta tem sido, há já algum tempo, objeto de estudo por vários autores [1-7].

Em trabalhos anteriores já publicados [5, 6], estudámos por HPLC-MS a composição dos extractos de folhas de murta obtidos através de diferentes técnicas, nomeadamente a extração supercritica, a extracção convencional e a extracção por ultrasons. Os resultados obtidos mostraram diferentes composições nos extratos obtidos, apresentando diferenças no número e no tipo de compostos nele presentes. Apenas o composto miricetina-ramnósido está presente em todos os extratos de folha de murta, independentemente da técnica de extracção usada para os obter [5-6]. Nos extratos obtidos por extração convencional, verifica-se que, além do composto referido, foram ainda identificados o ácido gálico, ácido elágico, miricetina, quercetina e quercetina-ramnósido, enquanto que no extrato obtido por ESC, além do já referido, foram ainda identificados a miricetina galactósido e a quercetina-ramnósido. Já nos extratos obtidos pela técnica de ultrassons foram identificados a quercetina-galactósido-galato, a miricetina-galactósido, a miricetina-galactósido-galato, e a miricetina [6].

No presente estudo analisou-se um extrato obtido também por ESC, mas usando um processo de adição de co-solvente mais simples, para investigar se esta alteração afetaria o número e tipo de compostos extraídos. Em vez de o etanol ser introduzido progressivamente na célula, ao longo do processo de extracção, utilizando uma bomba de líquidos, o volume total de etanol foi adicionado diretamente na célula de extração de uma só vez, após a introdução da amostra na célula.

\section{Material e Métodos}

\section{Material Vegetal}

Amostras de folhas de murta foram recolhidas na zona de Sintra (Portugal). Após a recolha, a planta foi identificada e depositada no Herbário do Instituto Superior de Agronomia da Universidade Técnica de Lisboa (co- 
ty of Lisbon (code: LISI 180/2006). The plant material was dried for two months out of the sunlight, and then sealed in black plastic bags and stored at $-20^{\circ} \mathrm{C}$.

\section{Standards and reagents}

Quercetin-3- $\beta$-D-glucoside, myricetin, myricetin3-O-rhamnoside, were purchased from Extrasynthese (Genay, France). Quinic acid was purchased from Sigma-Aldrich (Steinheim Germany). Absolute ethanol, methanol, acetonitrile, formic acid, all of the highest available purity, were purchased from Merck (Darmstadt, Germany). Carbon dioxide (N48-99.998\%) for supercritical extraction was supplied in cylinders by Air Liquide (Lisbon, Portugal).

\section{Extraction Method}

Supercritical extraction studies were performed with 30 $\mathrm{g}$ of ground myrtle leaves. The SFE apparatus in which the experiments were carried out is described elsewhere [7]. The experimental conditions used were: temperature $48^{\circ} \mathrm{C}$, pressure $10 \mathrm{MPa}$, SCF flow rate $130.71 \mathrm{dm}^{3}$ $\mathrm{h}^{-1}\left(0,238 \mathrm{~kg} \mathrm{~h}^{-1}\right)$, and $104 \mathrm{~cm}^{3}$ ethanol (co-solvent). This volume of ethanol was placed inside of the extraction vessel using a syringe.

\section{HPLC/MS Analysis}

The extracts were analysed in a LC-MS with a ProStar 410 autosampler, two 212-LC chromatography pumps, a ProStar 335 diode array detector and a 500-MS ion trap mass spectrometer with an electrospray ionisation (ESI) ion source (Varian, Palo Alto, CA, USA). Data acquisition and processing were performed using Varian MS Control 6.9 software. The phenolic compounds were separated in a Polaris column (Varian) RP18-A (150 $\mathrm{mm} \times 2 \mathrm{~mm}$ I.D., $5 \mu \mathrm{m}$ particle size) The column was kept at a controlled temperature $\left(35^{\circ} \mathrm{C}\right)$. The samples were injected into the column via a Rheodyne injector with a $20 \mu \mathrm{L}$ loop. The mobile phase consisted of water containing $0.1 \%$ formic acid (A) and acetonitrile containing $0.1 \%$ formic acid (B), at a flow rate of 250 $\mu \mathrm{L} / \mathrm{min}^{-1}$. The elution conditions were as follows: $7 \%$ B for $2 \mathrm{~min}$; 7-25\% B for $8 \mathrm{~min}$; $25-80 \%$ B for $35 \mathrm{~min}$; $80-100 \%$ B for $2 \mathrm{~min} ; 100 \%$ B for $3 \mathrm{~min} ; 100-7 \%$ B for $1 \mathrm{~min}$; and finally $7 \% \mathrm{~B}$ for $4 \mathrm{~min}$.. The mass spectra were acquired in the ESI negative and positive ion modes, in the range from $\mathrm{m} / \mathrm{z} 100$ to 1700 ; the optimized parameters were as follows: ion spray voltage, $\pm 4.9 \mathrm{kV}$; capillary voltage, 20 and $-60 \mathrm{~V}$; RF loading $90 \%$. Nitrogen was used as a nebulising and drying gas, at a pressure of 35 and 10 psi, respectively; drying gas temperature, $350{ }^{\circ} \mathrm{C}$. The multistage MS $\left(\mathrm{MS}^{n}\right)$ spectra were obtained with an isolation window of $2.0 \mathrm{Da}$, digo: LISI 180/2006). A planta foi seca durante dois meses, protegida da luz do sol, e em seguida foi selada e armazenada em sacos de plastico pretos, a $-20^{\circ} \mathrm{C}$.

\section{Padrões e reagentes}

Quercetina-3- $\beta$-D-glucósido, miricetina, miricetina-3-O-ramnósido, foram adquiridos na Extrasynthese (Genay, França). O ácido quinico foi comprado na Sigma-Aldrich (Steinheim Alemanha). Etanol absoluto, metanol, acetonitrilo, ácido fórmico, todos com o maior grau de pureza disponível, foram adquiridos na Merck (Darmstadt, Alemanha). O dióxido de carbono (N4899,998\%) utilizado na extração supercritica foi fornecido em cilindros pelo Ar Liquido (Lisboa, Portugal).

\section{Método de Extração}

Os ensaios de extração supercrítica foram realizados com $30 \mathrm{~g}$ de folhas de murta moídas. O aparelho ESC, onde os ensaios foram realizados já foi descrito noutra publicação [7]. As condições experimentais utilizadas foram: temperatura $48^{\circ} \mathrm{C}$, pressão $10 \mathrm{MPa}$, caudal FSC $130,71 \mathrm{dm}^{3} \mathrm{~h}^{-1}\left(0,238 \mathrm{~kg} \mathrm{~h}^{-1}\right)$ e $104 \mathrm{~cm}^{3}$ de etanol (co-solvente). Este volume de etanol foi colocado dentro da célula de extração usando uma seringa.

\section{Análise por HPLC/MS}

Os extratos foram analisados num LC-MS constituído por um ProStar 410 autosampler, duas bombas 212-LC de cromatografia, um detetor de matriz de díodos ProStar 335 e um espectrómetro 500-MS LC de massa de trapa de iões com uma fonte de iões de ionização por electrospray (ESI) equipado com um sistema Ion Trap (Varian, Palo Alto, CA, EUA). Realizou-se a aquisição e processamento de dados utilizando um software Varian MS Controlo 6,9.

As separações dos compostos fenólicos foram realizadas numa coluna Polaris (Varian) RP18-A (150 mm x 2 mm ID, tamanho de partícula $5 \mu \mathrm{m}$ ). A coluna foi mantida a uma temperatura controlada $\left(35^{\circ} \mathrm{C}\right)$. As amostras foram injetadas na coluna através de um injetor Rheodyne com um ciclo de $20 \mu \mathrm{L}$. A fase móvel lconsistiu em $0,1 \%(\mathrm{v} / \mathrm{v})$ de ácido fórmico em água $(\mathrm{A})$ e $0,1 \%$ (v/v) de ácido fórmico em acetonitrilo (B com um caudal de $0,25 \mathrm{~mL} \mathrm{~min}^{-1}$. As condições de eluição foram as seguintes:7\% de B durante 2 min; $7-25 \%$ B durante 8 min, 25-80\% B durante $35 \mathrm{~min} ; 80-100 \%$ B durante 2 min; 100\% B durante 3 min; 100-7\% B durante 1 min; e finalmente 7\% B durante 4 min.;. Os espectros de massa foram adquiridos em modo negativo e modo positivo num intervalo de m/z entre 100 e 1700; os parâmetros otimizados foram os seguintes: tensão de pulverização iónica, $\pm 4,9 \mathrm{kV}$; tensão capilar entre -20 a $-60 \mathrm{~V}$; carga 
excitation energy values of 1.0 and $2.5 \mathrm{~V}$ and an excitation time of $10 \mathrm{~ms}$. Data acquisition and processing were performed using Varian MS Control 6.9 software Identification of individual polyphenols was carried out using their retention times, and both spectroscopic and spectrometric data. Quantification of single polyphenols was directly performed by HPLC-DAD using a six-point regression curve built with the available standard of myricetin-3-O-rhamnoside. The regression equations and correlation coefficients were calculated using Microsoft Excel 2007 software with correlation coefficient values greater than 0.997 . The UV spectra for the unidentified compounds were compared with the available library of previously recorded UV spectra for a wide range of compounds.

\section{Results and Discussion}

The chromatographic analysis of the sample allowed the identification of seven phenolic compounds and the quantification of four of these compounds. The chromatograms obtained by HPLC-DAD-ESI-MS and respective mass spectra are shown in Figure 1.

Peak $1\left(R_{t}=3.7 \mathrm{~min}\right)$ was identified as quinic acid based on retention time and the absorbance spectrum by comparison with the standard. The identification was confirmed by MS/MS analysis of the deprotonated molecule [M-H] $]^{-}$at $m / z$ 191, which has produced the fragment ions $m / z 173,127,111,93,85$. Quinic acid has been identified in Myrtus communis L. extracts by Piras et al. [8]

Peak $2\left(\mathrm{R}_{\mathrm{t}}=8.5 \mathrm{~min}\right)$ exhibited a deprotonated molecule at $\mathrm{m} / \mathrm{z}$ 495, and its $\mathrm{MS}^{2}$ generated fragments at $\mathrm{m} / \mathrm{z} 343$ (loss of $152 \mathrm{Da}$, corresponding to a residue of gallic acid), $m / z 325$ (loss of $\mathrm{H}_{2} \mathrm{O}$ ), $\mathrm{m} / \mathrm{z} 191$ and 169, corresponding to quinic acid and gallic acid, respectively. By comparison with literature data [9], compound 2 was identified as quinic acid 3,5-di-O-gallate. The presence of this acid in Myrtus communis L. extracts was previously reported by Yoshimura et al. [10].

Peak $3\left(\mathrm{R}_{\mathrm{t}}=11.8 \mathrm{~min}\right)$ was identified as quinic acid 3,4,5-galloyl based in the mass spectrum showing one base peak at $m / z 647$, and $\mathrm{MS}^{2}$ fragments at $\mathrm{m} / \mathrm{z} 495$ (loss of $152 \mathrm{Da}$ ). The $\mathrm{MS}^{3}$ spectrum of $\mathrm{m} / \mathrm{z} 495$ showed as main fragments ions at $m / z 343,325,191,169$, following a fragmentation pattern similar to that of compound 2. This result indicated that both compounds belong to the same subclass of phenolic acids, being reported the presence of acids galloyl quinic acids result-
RF de 90\%. Utilizou-se azoto como gás de nebulização e de secagem, a uma pressão de 35 e 10 psi, respetivamente; temperatura do gás de secagem a $350^{\circ} \mathrm{C}$. Os espectros MS $\left(\mathrm{MS}^{\mathrm{n}}\right)$ multi-estágio foram obtidos com uma janela de isolamento de 2,0 $\mathrm{Da}$, valores de energia de excitação de 1,0 a $2,5 \mathrm{~V}$ e um tempo de excitação de $10 \mathrm{~ms}$. A identificação dos polifenóis individuais foi realizada usando os tempos de retenção e dados espectroscópicos e espectrométricos. A quantificação dos polifenóis foi realizada diretamente por HPLC-DAD usando uma curva de regressão construída com seis pontos de do padrão de miricetina-3-O-ramnósido. As equações de regressão e os coeficientes de correlação foram calculados usando o software Microsoft Excel 2007 com coeficientes de correlação superiores a 0.997. Os espetros de UV para os compostos não identificados foram comparados com a biblioteca de dados disponível.

\section{Resultados e Discussão}

A análise cromatográfica da amostra permitiu a identificação de sete compostos fenólicos e a quantificação de quatro desses compostos. Na Figura 1 apresentam-se os cromatogramas obtidos por HPLC-DAD-ESI/MS e os respetivos espetros de massa.

O pico $1\left(R_{t}=3,7 \mathrm{~min}\right)$ foi identificado como ácido quínico com base no tempo de retenção e espetro de absorvância por comparação com o padrão. A identificação foi confirmada por análise MS/MS da molécula desprotonada [M-H] $]^{-}$a $m / z 191$, que produziu os iões fragmento $\mathrm{m} / z 173,127,111,93,85$. O ácido quínico foi identificado em extratos de Myrtus communis por Piras et al. [8].

O pico $2\left(\mathrm{R}_{\mathrm{t}}=8,5 \mathrm{~min}\right)$ apresentou uma molécula desprotonada a $m / z 495$ e o seu $\mathrm{MS}^{2}$ gerou fragmentos a $m / z 343$ (perda de 152 Da correspondente a um resíduo de ácido gálico), $m / z 325$ (perda de $\mathrm{H}_{2} \mathrm{O}$ ), m/z 191 e 169, correspondentes ao ácido quínico e ao ácido gálico, respetivamente. Por comparação com dados da literatura [9], o composto 2 foi identificado como ácido quínico 3,5-di-O-galato. A presença deste ácido em extratos de Myrtus communis foi reportada por Yoshimura et al. [10].

O pico $3\left(\mathrm{R}_{\mathrm{t}}=11,8 \mathrm{~min}\right)$ foi identificado como ácido 3,4,5-galoilquínico com base no espetro MS que revelou um pico base a $m / z 647$ que nos ensaios $\mathrm{MS}^{2}$ originou o fragmento $\mathrm{m} / \mathrm{z} 495$ (perda de $152 \mathrm{Da}$ ). O espetro $\mathrm{MS}^{3}$ do ião selecionado $\mathrm{m} / \mathrm{z} 495$ mostrou picos a $\mathrm{m} / \mathrm{z}$ $343,325,191,169$, seguindo um padrão de fragmentação semelhante ao do composto 2 . Este resultado indicou que ambos os compostos pertencem à mesma sub- 


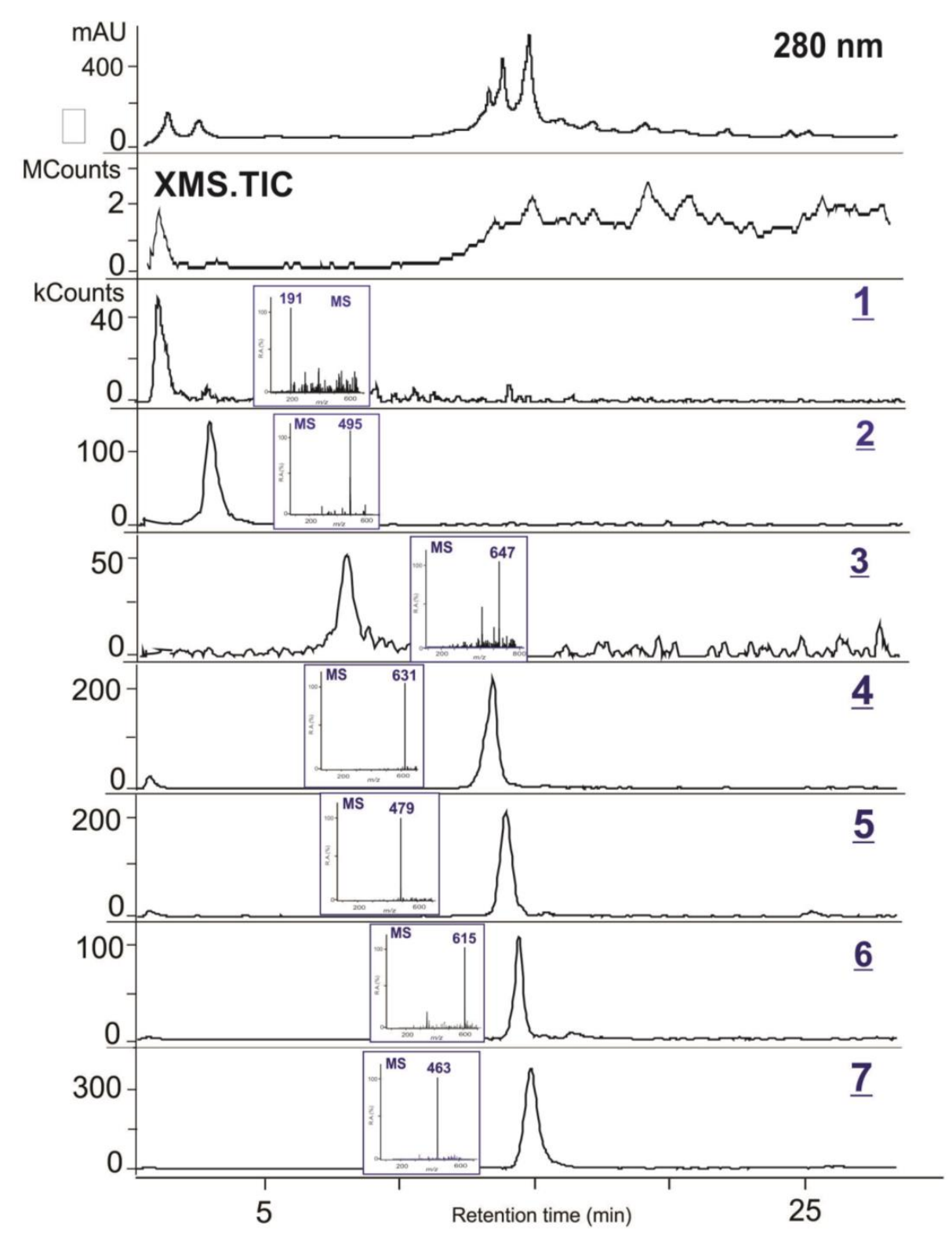

Figure 1/ Figura 1 - HPLC-DAD-MS chromatograms of extract of Myrtus communis L obtained by SFE

Cromatogramas HPLC-DAD-MS do extrato de Myrtus communis L. obtido por extração supercrítica.

ing from the esterification of one or more $\mathrm{OH}$ groups of the quinic acid with gallic acid. Compound $\mathbf{3}$ was assigned as a galloyl quinic acid derivative based on the fragmentation behaviour in the $\mathrm{MS}^{\mathrm{n}}$ experiments and by comparison with published data previously published.[11].

Peak $4\left(\mathrm{R}_{\mathrm{t}}=12.9 \mathrm{~min}\right)$ was identified as myricetin-galactoside-gallate based on its MS data. A standard was not available, thus the results were compared to the myricetin-rhamnoside standard. The MS spectrum revealed one base peak at $m / z 631$. The $\mathrm{MS}^{2}$ spectrum showed only one peak at $\mathrm{m} / \mathrm{z} 479$ due to the loss of a gallic classe de ácidos fenólicos, sendo reportado a presença de ácidos galoil-quínicos resultantes da esterificação de um ou mais grupos $\mathrm{OH}$ do ácido quínico com o ácido gálico. O composto 3 foi identificado como um derivado do ácido galoil-quínico baseado no comportamento de fragmentação nos ensaios de $\mathrm{MS}^{\mathrm{n}}$ e por comparação com dados publicados [11].O pico $4\left(\mathrm{R}_{\mathrm{t}}=12,9 \mathrm{~min}\right)$ foi identificado como miricetina-galactósido-galato com base nos dados de MS. O padrão não estava disponível, tendo os resultados sido comparados com o padrão miricetina-ramnósido. $\mathrm{O}$ espetro $\mathrm{MS}$ revelou um pico base a $m / z$ 631. O espetro $\mathrm{MS}^{2}$ apresentou apenas um pico 
acid residue (152 Da), corresponding to the cleavage of the ester of gallic acid. The $\mathrm{MS}^{3}$ spectrum exhibited two fragment ions, at $\mathrm{m} / \mathrm{z} 317$ and 316, assigned to the aglycone myricetin $\left(\mathrm{Y}_{0}^{-}\right)$and to the aglycone myricetin radical ion $\left(\mathrm{Y}_{0}^{-}-\mathrm{H}^{\bullet}\right)$, respectively. The formation of the aglycon is associated with the loss of $162 \mathrm{Da}$, indicating the presence of a glucose/galactose in the structure of the molecule. The $\mathrm{MS}^{4}$ spectrum of $\mathrm{m} / \mathrm{z} 317$ showed, as main fragments, ions at $\mathrm{m} / \mathrm{z} 289$ (CO loss), $179\left({ }^{1,2} \mathbf{A}_{0}\right.$. ), $151\left({ }^{1,2} \mathbf{A}_{0}{ }^{-}-\mathrm{CO}\right)$ and $137\left({ }^{1,2} \mathbf{B}_{0}{ }^{-}\right)$. These fragments are diagnostic for myricetin and confirmed the presence of a myricetin derivative. Based on published data by Romani et al. $[12,13]$, the sugar moiety linked to the myricetin was assigned as galactose.

Peak $5\left(\mathrm{R}_{\mathrm{t}}=13.6 \mathrm{~min}\right)$ was attributed to myricetin-galactoside. The UV spectrum showed two absorption bands (I and II) typical of a flavonol structure $(262 \mathrm{~nm}$ and $352 \mathrm{~nm}$ ). The MS spectra showed a peak at $\mathrm{m} / \mathrm{z} 479$ which fragmented in a pattern similar to that described for peak 4 , indicating the presence of a myricetin-glycoside [14].

Peak $6(\mathrm{Rt}=13.9 \mathrm{~min})$ was identified as quercetin-galactoside-gallate. The deprotonated molecule at $m / z 615$ produced a fragment ion at $\mathrm{m} / \mathrm{z} 463$ (loss of $152 \mathrm{Da}$ ) which, upon $\mathrm{MS}^{3}$ fragmentation, yielded the ion $\mathrm{m} / \mathrm{z}$ 301 (loss $162 \mathrm{Da}$ ) attributed to a quercetin aglycone. This assignment was confirmed through the further dissociation of ion $\mathrm{m} / \mathrm{z} 301$ that yielded typical fragment ions. The compound was described previously by Sannomiya et al.[15].

Peak $7\left(\mathrm{R}_{\mathrm{t}}=14.4 \mathrm{~min}\right)$ was attributed to myricetin-rhamnoside based on MS/MS data. The ion $\mathrm{m} / \mathrm{z} 463$, identified in the MS spectrum, upon fragmentation led to formation of aglycone at $\mathrm{m} / \mathrm{z} 317$. The $\mathrm{MS}^{3}$ spectrum originated the diagnostic ions of myricetin, $\mathrm{m} / \mathrm{z} 179$ $\left({ }^{1,2} \mathbf{A}_{0}^{-}\right), 151\left({ }^{1,2} \mathbf{A}_{0}^{-}-\mathrm{CO}\right), 137\left({ }^{1,2} \mathbf{B}_{0}^{-}\right)$. The results were confirmed by comparison with the standard of myricetin-rhamnoside and with the results of Arapitsas [16].

Table 1 summarizes the phenolic compounds identified by HPLC-ESI/MS ${ }^{\mathrm{n}}$ in the sample

Peaks 4, 5, 6 and 7 were quantified within the sample, as shown in Table 2.

In the extracts obtained by supercritical extraction with ethanol addition in the cell, two groups of phenolic compounds were identified, the galloyl quinic acid derivatives (quinic acid, 3,5-di-O-gallate quinic acid, 3,4,5-galloylquinic acid) and flavonoids glycosides (myricetin- galactoside gallate, quercetin-galactoside gallate, myricetin- galactoside, myricetin-rhamnoside). It should be noted that in this extract galloyl derivatives of quinic acids were identified which previously had not been identified in the extracts obtained by the other techniques studied (SFE with addition of co-solvent a m/z 479 atribuído à perda de um resíduo de ácido gálico (152 Da), correspondente à clivagem da ligação éster do ácido gálico. No espetro de massa $\mathrm{MS}^{3}$ foram identificados dois iões fragmento, $m / z 317$ e 316, atribuídos à aglicona $\left(\mathrm{Y}_{0}^{-}\right)$e ao ião radical aglicona da miricetina $\left(\mathrm{Y}_{0}^{-}-\mathrm{H}^{\bullet}\right)$, respetivamente. A formação da aglicona está associada à perda de $162 \mathrm{Da}$ indicando a presença de uma glucose/galactose na estrutura da molécula. O espetro $\mathrm{MS}^{4}$ do ião selecionado $\mathrm{m} / \mathrm{z} 317$ apresentou como fragmentos principais os iões $m / z 289$ (perda de CO), $179\left({ }^{1,2} \mathbf{A}_{0}^{-}\right), 151\left({ }^{1,2} \mathbf{A}_{0}^{-}-\mathrm{CO}\right)$ e $137\left({ }^{1,2} \mathbf{B}_{0}^{-}\right)$. Estes fragmentos são diagnósticos da miricetina e confirmaram a presença de um derivado da miricetina. Com base nos dados publicados por Romani et al. $[12,13]$ atribuiu-se uma galactose ao açúcar ligado à miricetina.

$\mathrm{O}$ pico $5\left(\mathrm{R}_{\mathrm{t}}=13,6 \mathrm{~min}\right)$ foi atribuído à miricetina-galactósido. O espetro UV apresentou duas bandas I e II de absorção características dos flavonóis (262 nm e 352 $\mathrm{nm})$. O espetro MS apresentou um pico a $\mathrm{m} / \mathrm{z} 479$ que se fragmentou seguindo um padrão idêntico ao descrito para o pico 4 indicando a presença de uma miricetina-glicósido (Singh et al.) [14].

O pico $6 \quad\left(R_{t}=13,9 \mathrm{~min}\right)$ foi identificado como quercetina-galactósido-galato. A molecula desprotenada $\mathrm{m} / \mathrm{z} 615$ mostrou apenas um pico a $\mathrm{m} / \mathrm{z} 463$ (perda de $152 \mathrm{Da}$ ) que ao fragmentar-se $\mathrm{MS}^{3}$ originou o ião $\mathrm{m} / \mathrm{z} 301$ (perda $162 \mathrm{Da}$ ), atribuido à aglicona desprotonada da quercetina. . Esta atribuição foi confirmada através da dissociação adicional do íon $\mathrm{m} / \mathrm{z} 301$ que produziu os iões fragmentos típicos. O composto foi descrito anteriormente por Sannomiya et al. [15].

$\mathrm{O}$ pico $7\left(\mathrm{R}_{\mathrm{t}}=14,4 \mathrm{~min}\right)$ foi atribuído à miricetina-ramnósido com base nos dados MS/MS. O ião $\mathrm{m} / z 463$ identificado no espetro MS ao fragmentar-se levou à formação da aglicona a $\mathrm{m} / \mathrm{z} 317$. O espetro $\mathrm{MS}^{3}$ originou os iões diagnósticos da miricetina, $m / z 179\left({ }^{1,2} \mathbf{A}_{0}\right), 151\left({ }^{1,2} \mathbf{A}_{0}{ }^{-}\right.$ $\mathrm{CO}), 137\left({ }^{1,2} \mathbf{B}_{0}^{-}\right)$. Os resultados foram confirmados por comparação com os do padrão miricetina-ramnósido e com resultados de Arapitsas [16].

$\mathrm{Na}$ Tabela 1 sumarizam-se os compostos fenólicos identificados por HPLC-ESI/MS ${ }^{\mathrm{n}}$ para a amostra. Os picos 4,5,6 e 7 desta amostra foram quantificados, conforme apresentados na tabela 2.

Nos extratos obtidos por extração supercrítica com adição de etanol na célula, foram identificados dois grupos de compostos fenólicos, os derivados dos ácidos galoil-quínicos (ácido quínico, ácido quínico 3,5-di-O-galato, ácido 3,4,5-galoilquínico) e os flavonóides glicosídeos (miricetina-galactósido-galato, quercetina-galactósido-galato, miricetina-galactósido, miricetina-ramnósido). De salientar que neste extrato foram identificados derivados de ácidos galoilquínico que não tinham sido 
Table 1 / Tabela 1 - HPLC-DAD-MS/MS identification of phenolics compounds in extracts of Myrtus communis L. obtained by SFE Identificação de compostos fenólicos por HPLC-DAD-MS/MS no extrato de Myrtus communis L obtido por extração supercrítica.

\begin{tabular}{|c|c|c|c|c|}
\hline $\begin{array}{l}\text { Peaks } \\
\text { Picos/ }\end{array}$ & $\mathbf{R}_{\mathrm{t}}(\min )$ & $\begin{array}{c}{[\mathrm{M}-\mathrm{H}]^{-}} \\
m / \mathbf{z}\end{array}$ & $\begin{array}{l}M^{n}{ }^{n} \text { fragment } m / z \text {, ion } \\
M S^{n} \text { ião fragmento } m / z\end{array}$ & $\begin{array}{l}\text { Probable compound } \\
\text { Composto provável }\end{array}$ \\
\hline 1 & 3.7 & 191 & $\begin{array}{l}\mathrm{MS}^{2}[191]: 173\left[\mathrm{M}-\mathrm{H}-\mathrm{H}_{2} \mathrm{O}\right]^{-}, 127\left[\mathrm{M}-\mathrm{H}-\mathrm{H}_{2} \mathrm{O}\right]^{-}, 111 \\
{\left[\mathrm{C}_{6} \mathrm{H}_{7} \mathrm{O}_{2}\right]^{-} 93\left[\mathrm{C}_{6} \mathrm{H}_{5} \mathrm{O}\right]^{-}, 85\left[\mathrm{C}_{4} \mathrm{H}_{5} \mathrm{O}_{2}\right]^{-}}\end{array}$ & Quinic acid / Ácido quínico \\
\hline 3 & 11.8 & 647 & $\begin{array}{l}\mathrm{MS}^{2} \text { [647]: } 495 \text { [M-H-152] } \\
\mathrm{MS}^{3}[495]: 343 \text { [M-H-2x152], } 325 \text { [M-H-(2x152- } \\
\left.\left.\mathrm{H}_{2} \mathrm{O}\right)\right]^{-}, 191 \text { (quinic acid/ácido quínico), } 169 \text { (gallic } \\
\text { acid/ácido gálico) }\end{array}$ & $\begin{array}{l}\text { 3,4,5-galloylquinic acid/ } \\
\text { Ácido 3,4,5-galoilquínico }\end{array}$ \\
\hline 5 & 13.6 & 479 & $\begin{array}{l}\mathrm{MS}^{2}[479]: 317 \mathrm{Y}_{0}^{-}, 316\left[\mathrm{Y}_{0}^{-}-\mathrm{H}^{-}\right. \\
\mathrm{MS}^{3}[317]: 289 \mathrm{Y}_{0}^{-}-\mathrm{CO}^{-}, 179^{1,2} \mathbf{A}_{0}^{-}, 151\left({ }^{1,2} \mathbf{A}_{0}^{-}-\mathrm{CO}\right) \\
137^{1,2} \mathbf{B}_{0}^{-}\end{array}$ & $\begin{array}{l}\text { Myricetin galactoside/ } \\
\text { Miricetina-galactósido }\end{array}$ \\
\hline 6 & 13.9 & 615 & $\begin{array}{l}\mathrm{MS}^{2}[615]: 463[\mathrm{M}-\mathrm{H}-152]^{-} \\
\mathrm{MS}^{3}[463]: 301 \mathrm{Y}_{0}^{-}\end{array}$ & $\begin{array}{l}\text { Quercetin galactoside- } \\
\text { gallate/ Quercetina- } \\
\text { galactósido-galato }\end{array}$ \\
\hline
\end{tabular}

Table 2 / Tabela 2 - Quantitative analysis of the major phenolic compounds identified in the extracts obtained by SFE Quantificação dos principais compostos fenólicos identificados no extracto obtido por extração supercritica.

\begin{tabular}{cccc}
\hline $\begin{array}{c}\text { Peaks/ } \\
\text { Picos }\end{array}$ & Compounds/Compostos & $\begin{array}{c}\text { Concentration/ } \\
\text { Concentração } \\
\left(\mathbf{m g ~ m L}^{-1}\right)\end{array}$ & $\begin{array}{c}\text { Concentration/ } \\
\text { Concentração } \\
\left(\mathbf{m g ~ g}^{-1} \text { plant/planta) }^{-}\right.\end{array}$ \\
\hline 4 & $\begin{array}{c}\text { Myricetin galactoside-gallate/ } \\
\text { Miricetina-galactósido-galato } \\
\text { Myricetin galactoside/ } \\
\text { Miricetina-galactósido }\end{array}$ & $1.08 \mathrm{E}-01$ & $2,73 \mathrm{E}-03$ \\
5 & $\begin{array}{c}\text { Quercetin galactoside-gallate/ } \\
\text { Quercetina-galactósido-galato } \\
\text { Myricetin rhamnoside } \\
6\end{array}$ & $2.81 \mathrm{E}-01$ & $7,14 \mathrm{E}-03$ \\
7 & Miricetina-ramnósido & $2.41 \mathrm{E}+01$ & $6,12 \mathrm{E}-01$ \\
\hline
\end{tabular}


with liquid pump, conventional extraction and ultrasound extraction).

Regarding the technique used in this study, Lang and Wai [17] state that the introduction of ethanol directly into the cell is the most simple and inexpensive technique of co-solvent addition. However, the authors point out that some care is required in the use of this technique, as it is necessary to ensure that the binary fluid is in supercritical state. Additionally, the potential exists that the co-solvent could be pushed out of the cell at the beginning of the dynamic extraction, which would produce inconsistent results and increase the number of necessary repetitions. In the present case, the ejection of ethanol at the beginning of the extraction apparently did not affect the fluid's extraction ability, as it produced an extract with a larger number of identifiable compounds than with the other techniques previously studied.

\section{Conclusion}

The results obtained showed that the composition of the extract obtained, using the method of direct introduction of co-solvent inside the extraction cell, is different from the compositions previously obtained. An extract with different and greater numbers of compounds and was obtained. However, since the conditions of pressure and temperature used were not identical to those of other essays of SFE, it is not possible to conclude that this technique, more simple and inexpensive, is superior to one utilising a liquid pump. To this end, it will still be necessary that these tests are repeated under the conditions of pressure and temperature used in previous tests.

\section{Conflict of interests}

The author declares that there is no personal or financial relationship that can be understood as presenting a potential conflict of interest. identificados nos extractos obtidos pelas outras técnicas estudadas anteriormente (ESC com adição de co-solvente com bomba de líquidos, extracção convencional e extração por ultrassons) $[5,6]$.

Relativamente à técnica usada, Lang e Wai [17] referem que a introdução do etanol diretamente na célula é a técnica mais simples de adição de co-solvente e a mais económica. No entanto, o autor refere que é necessário algum cuidado na utilização desta técnica, pois é necessário assegurar que o fluido binário se encontra no estado supercrítico, e também porque pode acontecer que o co-solvente seja empurrado para fora da célula logo no início da extração dinâmica, o que tornaria os resultados inconsistentes, e obrigando a fazer várias repetições. No caso presente, a ejecção de etanol no início da extração aconteceu de facto mas, aparentemente, sem que isso afectasse a capacidade de extracção, já que foi recolhido um extracto com um maior número de compostos do que com as outras técnicas anteriormente estudadas $[5,6]$.

\section{Conclusão}

Os resultados obtidos permitiram verificar que a composição do extrato obtido usando o método de introdução direta do co-solvente no interior da célula de extração é diferente das composições anteriormente obtidas. Obteve-se um extrato com compostos diferentes e em maior número. No entanto, uma vez que as condições de pressão e temperatura utilizadas não foram idênticas às de outros ensaios de ESC, não é possível concluir ainda pela superioridade desta técnica, mais simples e barata, relativamente à que utiliza a bomba de líquidos. Para tal, será ainda necessário que estes ensaios sejam repetidos nas condições de pressão e temperatura utilizadas em ensaios anteriores.

\section{Conflito de interesses}

O autor declara não existir qualquer relação pessoal ou financeira que possa ser entendida como representando um potencial conflito de interesses. 


\section{References / Referências}

1. Tuberoso CIG, Barra A, Angioni A, Sarritzu E, Pirisi F. 2006. Chemical Composition of Volatiles in Sardinian Myrtle (Myrtus communis L.) Alcoholic Extracts and Essential Oils. Journal of Agricultural and Food Chemistry 54, 1420-1426.

2. Wannes A, Mhamdi B, Marzouk B. 2009. GC Comparative Analysis of Leaf Essential Oils from Two Myrtle Varieties at Different Phenological Stages. Chromatographia 69, 145-150.

3. Jamoussi B, Romdhane M, Abderraba A, Hassine B, Gadri A. 2005. Effect of harvest time on the yield and composition of Tunisian myrtle oils. Flavour and Fragrance J. 20, 274-277.

4. Özek T, Demirci B, Baser K. 2000. Chemical Composition of Turkish Myrtle Oil. Journal of Essential Oil Research, 12, 541-544

5. Pereira P, Cebola MJ, Oliveira MC, Bernardo-Gil MG. 2016. Supercritical fluid extraction vs conventional extraction of myrtle leaves and berries: comparison of antioxidant activity and identification of bioactive compounds. J Supercrit Fluid 113, 1-9.

6. Pereira P, Cebola MJ, Oliveira MC, Bernardo-Gil MG. 2017. Antioxidant Capacity and Identification of Bioactive Compounds of Myrtus communis L. Extract Obtained by Ultrasound-Assisted Extraction. J Food Sci Tech DOI:10.1007/s13197-017-2907-y

7. Pereira P, Bernardo-Gil MG, Cebola MJ, Maurício E, Romano A. 2013. Supercritical fluid extracts with antioxidant and antimicrobial activities from myrtle (Myrtus communis L.) leaves. Response surface optimization. J Supercrit Fluid 83, 57-64.

8. Piras FM, Dettori MF, Magnani A. 2009. ToF-SIMS PCA analysis of Myrtus communis L. Applied Surface Science, 255, $7805-7811$.

9. Del Rio D, Stewart AJ, Mullen W, Burns J, Lean MEJ, Brighenti F, Crozier A. 2004. HPLC-MSn Analysis of Phenolic Compounds and Purine Alkaloids in Green and Black Tea. Journal of Agricultural and Food Chemistry, 52 (10) 2807-15.

10. Yoshimura M, Amakura Y, Tokuhara M, Yoshida T. 2008. Polyphenolic compounds isolated from the leaves of Myrtus communis. Journal of Natural Medicines 63, 366-368.

11. Moore JP, Westall KL, Ravenscroft N, Farrant JM, Lindsey GG, Brandt WF. 2005. The predominant polyphenol in the leaves of the resurrection plant Myrothamnus flabellifolius, 3,4,5 tri-O-galloylquinic acid, protects membranes against desiccation and free radical-induced oxidation. Biochemical Journal, 385 (1), 301-308.

12. Romani A, Campo M, Pinelli P. 2012. HPLC/DAD/ESI-MS analyses and anti-radical activity of hydrolyzable tannins from different vegetal species. Food Chemistry 130, 214-221.

13. Romani A, Pinelli P, Mulinacci N, Vincieri FF, Tattini M. 1999. Identification and Quantification of Polyphenols in Leaves of Myrtus communis L. Chromatographia $49(1 / 2), 17-22$.

14. Singh AP, Luthria D, Wilson T, Vorsa N, Singh V, Banuelos GS, Pasakdee S. 2009. Polyphenols content and antioxidant capacity of eggplant pulp. Food Chemistry 114, 955-961.

15. Sannomiya M, Montoro P, Piacente S, Pizza C, Brito AR, Vilegas W. 2005. Application of liquid chromatography/electrospray ionization tandem mass spectrometry to the analysis of phenolics compounds from an infusion of Byrsonima crassa Niedenzu. Rapid Communication in Mass Spectrometry 19 (16), 2244-2250.

16. Arapitsas P. 2008. Identification and quantification of polyphenolics compounds from okra seeds and skins. Food Chemistry 110 (4), $1041-1045$.

17. Lang Q. e Wai CM. 2001. Supercritical fluid extraction in herbal and natural product Studies - a practical review. Talanta, 53, $771-782$. 